\title{
TALIF Measurements of Atomic Nitrogen in the Afterglow of a Nanosecond Capillary Discharge
}

\author{
T. L. Chng ${ }^{1}$, N. D. Lepikhin ${ }^{1, *}$, I. S. Orel ${ }^{1}$, N. A. Popov ${ }^{3}$ and S. M. Starikovskaia ${ }^{1}$ \\ ${ }^{1}$ Laboratory of Plasma Physics (CNRS, Ecole Polytechnique, Sorbonne Universities, University \\ Paris-Sud), Institut Polytechnique de Paris, route de Saclay, 91128 Palaiseau, France \\ ${ }^{2}$ Skobeltsyn Institute of Nuclear Physics, Moscow State University, Moscow, 119991, Leninsky \\ gory, Russia
}

\begin{abstract}
The atomic nitrogen $(\mathrm{N})$ density in a nanosecond pulse capillary discharge is measured using two-photon laser induced fluorescence (TALIF). The capillary discharge is favored for its unique combination of both large reduced fields $(E / N)$ and high specific deposited energies. Under such conditions, we find that a pure nitrogen $\left(\mathrm{N}_{2}\right)$ capillary discharge at a pressure of $27 \mathrm{mbar}$ and temperature of about $300 \mathrm{~K}$, produces a peak $\mathrm{N}$-atom density of $1.29 \cdot 10^{17} \mathrm{~cm}^{-3}$, corresponding to an extremely high dissociation degree of about $10 \%$. The time evolution of the $\mathrm{N}$-atom density is tracked from a few hundred ns after discharge initiation, up to several ms when the concentration of $\mathrm{N}$-atoms falls below the detection limit. The temporal evolution curve exhibits a trapezoidal-like shape, characterized by an initial rise in the $\mathrm{N}$-atom density up to a few $\mu \mathrm{s}$, followed by a relatively flat and constant profile until about $1 \mathrm{~ms}$, and finally terminating with a drop to near detection limits at about $10 \mathrm{~ms}$. The high electron densities $\left(\approx 10^{15} \mathrm{~cm}^{-3}\right)$ and efficient production of electronically excited states associated with this type of discharge is found to have a profound effect on the consequent kinetics. A process of stepwise dissociation through electron impact of the $\mathrm{N}_{2}\left(\mathrm{~A}^{3} \Sigma_{\mathrm{u}}^{+}, \mathrm{B}{ }^{3} \Pi_{\mathrm{g}}, \mathrm{C}^{3} \Pi_{\mathrm{u}}\right)$ excited states is examined and proposed as a possible explanation for the unusually high energy efficiency of $\mathrm{N}$-atom production. The present study shows that the capillary discharge is an extremely effective source of $\mathrm{N}$-atoms, and forms the impetus for continued study of discharges with both high levels of specific deposited energies ( $\geq 1 \mathrm{eV} /$ molecule) and reduced electric fields ( $E / N \geq 150 \mathrm{Td}$ ).
\end{abstract}

\footnotetext{
* Current affiliation: Ruhr University Bochum, Faculty of Physics and Astronomy, Institute for Plasma and Atomic Physics, Germany
} 


\section{Introduction}

Over the past decade, plasmas produced by nanosecond pulses has evolved into an enabling technology across numerous disciplines spanning aerodynamics [1,2], combustion [3-5], biology and medicine [6,7]. The principal mechanism behind such plasmas is that the application of high voltage pulses with a rapid (nanosecond) rise time and short pulse width (tens to hundreds of nanoseconds), creates a system of strong nonequilibrium, allowing access to voltages significantly exceeding the DC breakdown threshold [8]. At extremely high $\left(\sim 10^{3} \mathrm{Td}\right)$ electric fields in the front of a nanosecond discharge, the spatial uniformity of these plasmas can be maintained, from moderate pressures (tens of mbar) $[9,10]$ up to atmospheric pressure $[11,12]$. The discharge propagates in the form of a Fast lonization Wave (FIW), leaving in its wake a plasma with still relatively high electric fields $(200-400 \mathrm{Td})$, sustained throughout the duration of the high-voltage pulse. These fields efficiently excite electronic levels of atoms and molecules [13], which can consequently lead to molecular dissociation, providing one of the most efficient chemical pathways in nanosecond discharges.

High chemical reactivity of nanosecond plasmas via dissociation has only recently been experimentally demonstrated and confirmed by numerical modeling. High dissociation degrees of oxygen molecules in a pin-to-pin discharge in air at atmospheric pressure [14-17], and in a capillary discharge at tens of mbar [18] was obtained using high voltage pulse durations on the order of tens of nanoseconds. The efficiency of molecular oxygen dissociation has been attributed in [19] to dissociation via direct electron impact in the discharge (via A ${ }^{3} \Sigma_{\mathrm{u}}^{+}, \mathrm{C}^{3} \Delta_{\mathrm{u}}, \mathrm{c}{ }^{1} \Sigma_{\mathrm{u}}^{-}$and $\mathrm{B}^{3} \Sigma_{\mathrm{u}}^{-}$excited $\mathrm{O}_{2}$ states) and through collisional dissociation with electronically excited molecular nitrogen during the discharge and nearest afterglow, $\mathrm{N}_{2}{ }^{*}+\mathrm{O}_{2} \rightarrow \mathrm{N}_{2}\left(\mathrm{X}^{1} \Sigma_{\mathrm{g}}^{+}, \mathrm{v}\right)+\mathrm{O}\left({ }^{3} \mathrm{P}\right)+\mathrm{O}\left({ }^{3} \mathrm{P},{ }^{1} \mathrm{D}\right)$, where $\mathrm{N}_{2}$ * is one of the following states: $\mathrm{N}_{2}\left(\mathrm{~A}^{3} \Sigma_{\mathrm{u}}^{+}, \mathrm{v}\right), \mathrm{N}_{2}\left(\mathrm{~B}^{3} \Pi_{\mathrm{g}}\right), \mathrm{N}_{2}\left(\mathrm{a}^{\prime}{ }^{1} \Sigma_{\mathrm{u}}^{-}\right), \mathrm{N}_{2}\left(\mathrm{C}^{3} \Pi_{\mathrm{u}}\right)$.

Nanosecond capillary discharges, by virtue of their small volume, are an interesting subset of nanosecond plasmas, in that they provide a configuration where the specific energy deposited into the system is very high (a few $\mathrm{eV} /$ molecule). At such high values of deposited energy, the plasma chemistry is dominated by interactions between excited state species rather than with the bath gas. Previous studies have characterized important parameters of the discharge such as the gas temperature, electric field and electron density at moderate pressures around several tens of mbar for both pure $\mathrm{N}_{2}$ and $\mathrm{N}_{2}: \mathrm{O}_{2}[20]$. This has been supplemented by spectroscopic measurements of excited state species and atomic species such as O-atoms [18].

Most of the current work indicates that it is extremely difficult to achieve a high dissociation degree of $\mathrm{N}_{2}$ molecules in a gas-discharge nitrogen plasmas. However, in [35], the authors were able to obtain a very high fraction of nitrogen dissociation in a lowpressure ICP discharge at $P<100$ mTorr in pure nitrogen and in $\mathrm{N}_{2}: A r$ mixtures. The 
actinometry technique was used to quantitatively determine the nitrogen dissociation degree. A dissociation degree of up to $70 \%$ was obtained at high discharge power, and thus at high electron densities. Judging from the linear dependence of the dissociation degree upon power of the discharge [35], the energy efficiency is not expected to change significantly.

Measurements of $\mathrm{N}$-atom density in the $\mathrm{DC}$ discharge in pure nitrogen at $P=5-40$ Torr in the range of reduced electric fields $E / N=40-100 \mathrm{Td}$ are presented in [44]. The

authors show that the energy efficiency of nitrogen dissociation can be significantly increased in the presence of vibrational excitation of $\mathrm{N}_{2}$, but even then, the efficiency of $\mathrm{N}$-atom production did not exceed $0.9 \mathrm{~N}$-atoms per $100 \mathrm{eV}$ of deposited energy.

The main objective of this study is to examine the $\mathrm{N}$-atom density and energy efficiency in a moderate pressure nanosecond capillary discharge, with the aim of understanding the kinetics of discharges with high specific energy coupling and reduced electric field, and to support related modeling efforts.

\section{Experimental Setup}

\subsection{Discharge specifics}

A schematic of the discharge cell used in this work is presented in figure 1 . This configuration is very similar to that used in $[18,20]$, with slight differences in the dimensions of the discharge tube and the interelectrode distance. The tube is a cylindrical quartz capillary with a nominal length of $85 \mathrm{~mm}$ and an internal and external diameter of $1.7 \mathrm{~mm}$ and $3.0 \mathrm{~mm}$ respectively. Two pin-shaped brass electrodes, each with a diameter of $1 \mathrm{~mm}$ tapering to a tip radius of about $100 \mu \mathrm{m}$, are inserted at both ends along the length of the capillary, giving rise to an inter-electrode gap of about $67 \mathrm{~mm}$.

Pure $\mathrm{N}_{2}$ gas enters and exits the capillary via two glass side arms located along the length of the capillary, and at a flow rate of $5 \mathrm{sccm}$ by means of a Brooks Instruments 5850TR mass flow controller. This flow rate is chosen to ensure total gas renewal before each successive high voltage pulse. The static pressure in the middle of the capillary was maintained at 27 mbar based on previous work which indicated this as a good operating pressure for initiating a stable plasma while maximizing the energy coupled to the plasma. Pressure measurements are made using a pair of CMR 362 capacitive pressure sensors (from Pfeiffer Vacuum SAS), each positioned at an approximately equidistant location upstream and downstream of the capillary. The static pressure is determined by taking the average of both these sensor readings. This approach for pressure quantification is chosen due to difficulties associated with directly mounting a pressure transducer onto the capillary and has been validated numerically using a Navier-Stokes solver [18]. 


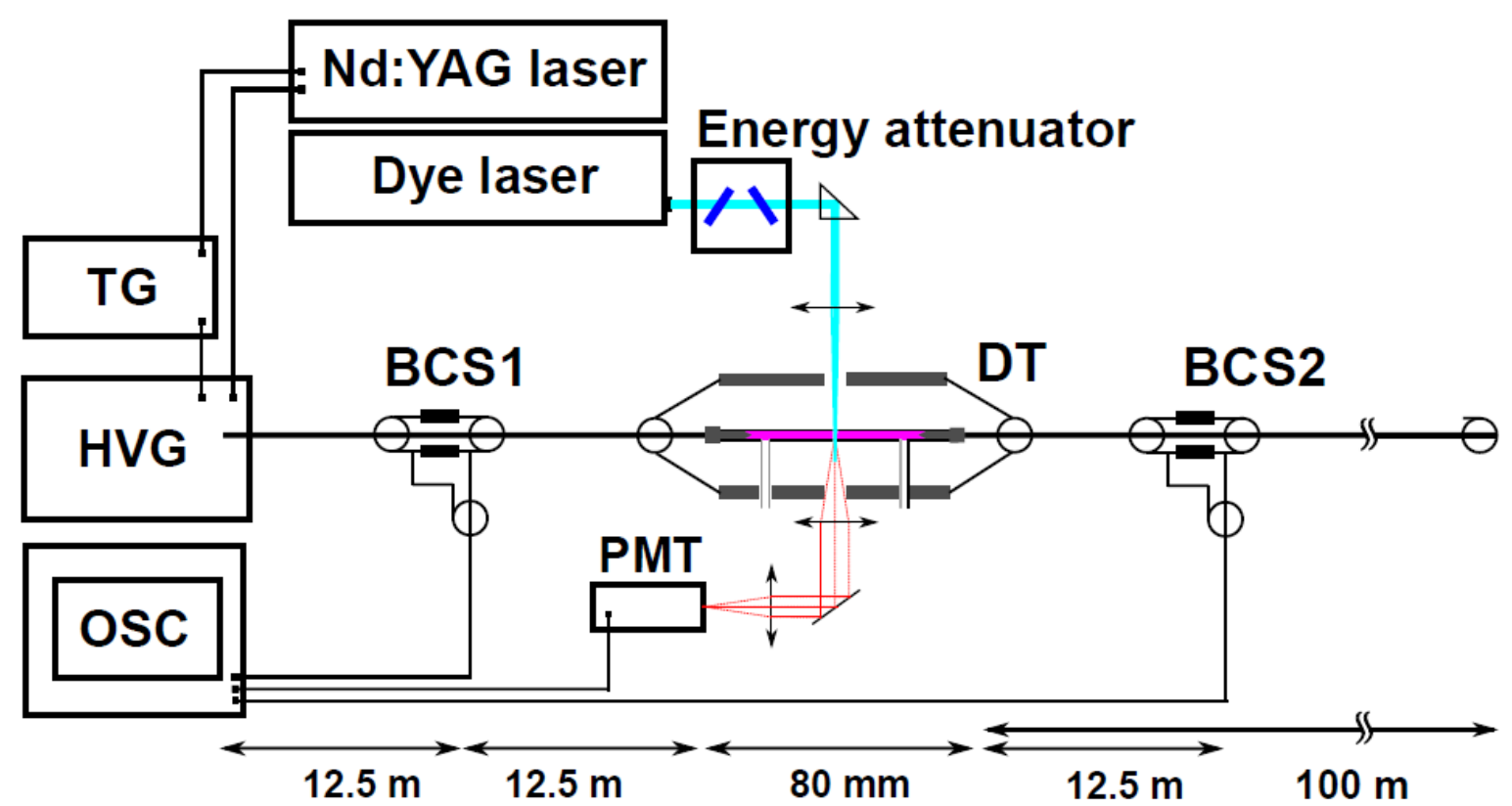

Figure 1. Schematic of the experimental setup, comprising the discharge cell and the optical equipment and components for the TALIF measurements.

High voltage nanosecond pulses are delivered by a FPG 12-1NM high-voltage generator (HVG) manufactured by FID GmbH. Positive polarity, $9.3 \mathrm{kV}$ amplitude voltage waveforms with a rise time of $4 \mathrm{~ns}$ and a full width at half maximum (FWHM) of $30 \mathrm{~ns}$ are produced by this HVG at a repetition rate of $10 \mathrm{~Hz}$. The output of this HVG is connected to one of the electrodes (high-voltage electrode) via a $50 \Omega$ impedance, RG213, $25 \mathrm{~m}$ coaxial cable. A $100 \mathrm{~m}$ length coaxial cable is connected to the second (low-voltage) electrode but with its other end short circuited. The central wires of each of the coaxial cables are electrically connected to the electrodes, while the respective shielding in each cable is connected to a rectangular $48 \mathrm{~mm}$ by $67 \mathrm{~mm}$ metallic screen which encapsulates the capillary. The installation of the capillary and grounded screen may therefore be treated as a break within a long coaxial cable.

Due to an impedance mismatch between the coaxial cable and the discharge cell (viz. electrical load), each pulse delivered by the HVG is reflected two times, once from the high-voltage electrode and a second time from the HVG itself. This entire process repeats itself only twice, limited by the internal circuitry of the HVG, and leads to a sequence of 3 incident pulses delivered to the discharge cell. Each incident pulse is separated from the next by about $250 \mathrm{~ns}$, corresponding to the round trip propagation time of the high-voltage pulse within the coaxial cable (see figure 2).

The high voltage waveforms are measured by means of two custom-made, calibrated back current shunts (BCS), embedded within each of the coaxial cables connected to the high and low voltage electrodes. The BCS installed before the high-voltage electrode 
(BCS1) captures the incident and reflected waveforms from the cell, while the second BCS located after the low-voltage electrode (BCS2) is responsible for detecting the transmitted voltage waveforms. It should be noted that a substantial body of work has been devoted to the development and use of these BCSs as a means of measuring the electrical current and energy coupled to a discharge [21,22]. The electrical signals from the BCS, along with the TALIF signals described in more detail below, are recorded via a LeCroy WaveRunner 64Xi-A oscilloscope with a bandwidth of $600 \mathrm{MHz}$ and a sampling rate of $5 \mathrm{GS} / \mathrm{s}$ (i.e. 200 ps per time step).
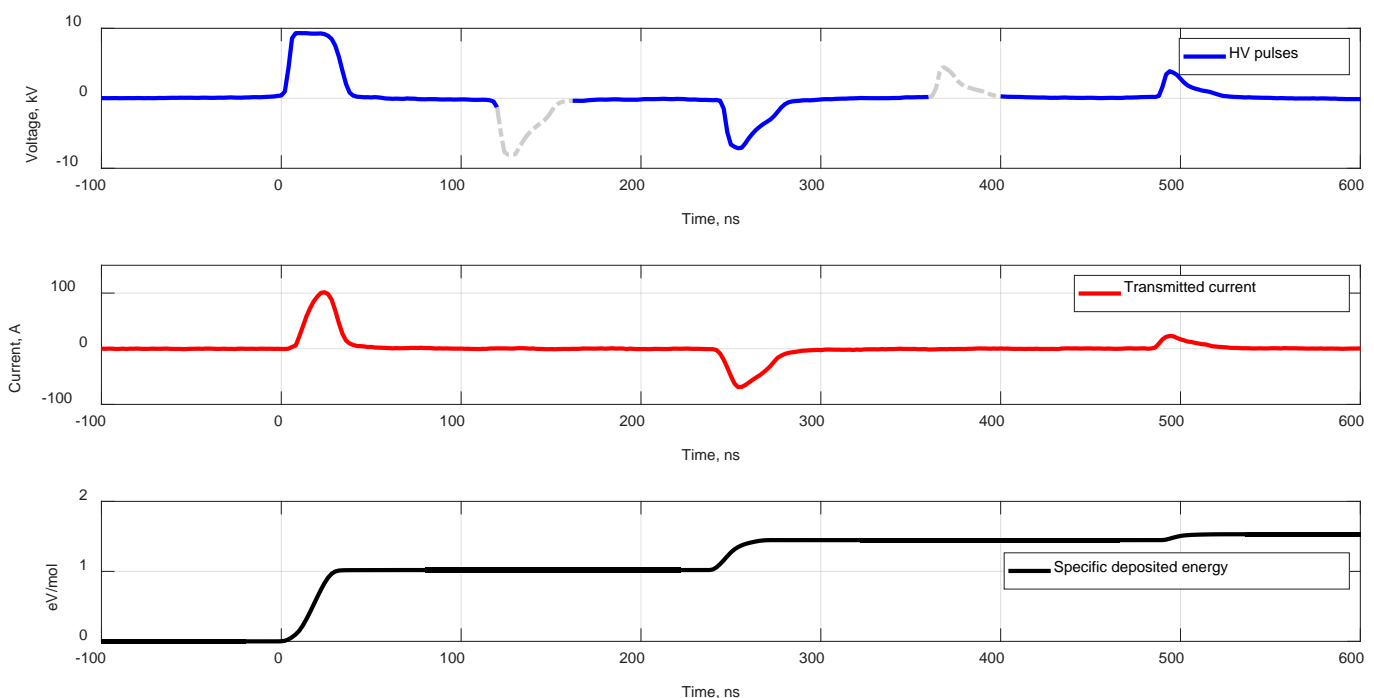

Figure 2. Waveforms showing the high-voltage pulses measured by BCS1 (incident pulses are shown in blue while reflected pulses are colored in gray dotted lines), transmitted current (BCS2) and the specific deposited energy.

\subsection{TALIF measurements of atomic nitrogen}

Two-photon absorption laser-induced fluorescence (TALIF) is used to track the time evolution of the atomic nitrogen density in the discharge. The laser system used for the TALIF experiments consists of a Sirah Lasertechnik Cobra Stretch dual-grating dye laser driven by a frequency-doubled Quanta Ray Nd:YAG laser. A dye-mix of Rhodamine B and Rhodamine 101 (supplied by Sirah Lasertechnik) is employed as the lasing medium, and pumped with $532 \mathrm{~nm}$ light to produce output within the visible range of $621 \mathrm{~nm}$, at a repetition rate of $10 \mathrm{~Hz}$. This light is then frequency-tripled via two successive alpha-BBO non-linear crystals, to yield $3 \mathrm{~mJ}$, tunable UV pulses near $206 \mathrm{~nm}$ with a FWHM pulse duration of about $6.5 \mathrm{~ns}$.

As illustrated in figure 1, this UV light is focused with a 1 inch diameter, $50 \mathrm{~cm}$ focal length plano-convex lens, propagating along an axis orthogonal to the length of the 
capillary and at a distance approximately halfway between the two electrodes. In addition, a high precision translational lens mount (Thorlabs LM1XY/M) ensures that the laser beam intersects well with the centerline of the capillary cross-section.

For the TALIF experiments, the laser intensity is appropriately reduced to a value of between $10-300 \mu \mathrm{J}$ through two mutually compensating, near-Brewster's angle tilt plates. The amount of energy reduction can be precisely controlled by small angular adjustments to the tilt plates, while leaving the temporal profile of the transmitted light unchanged. Furthermore, to avoid damaging the capillary, the laser intensity is attenuated by locating the beam focus slightly behind the capillary. The laser intensity is monitored with a 1 ns rise time photodiode (Thorlabs DET10A) by picking off a tiny fraction of the main beam with a quartz plate. The integrated photodiode signal exhibits a $\pm 5 \%$ correlation with the energy measured by a Newport 919P-003-10 thermopile power meter.
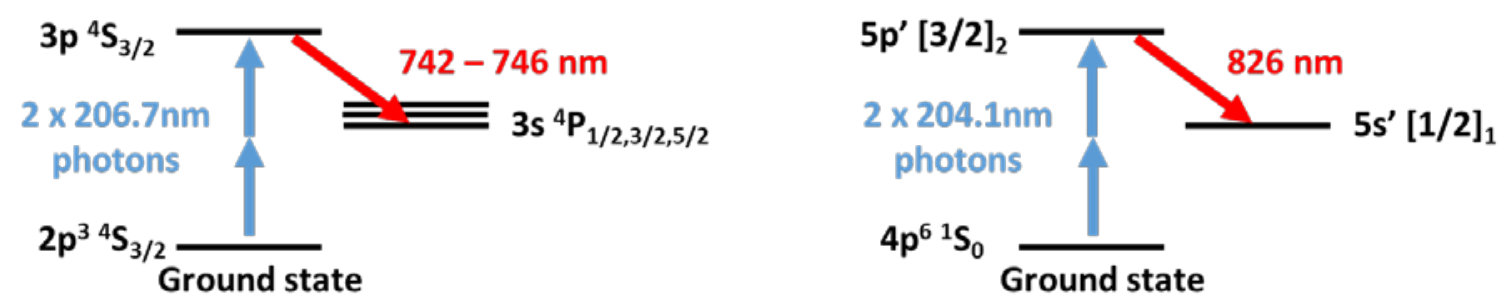

Figure 3. Two photon excitation scheme used for a) atomic nitrogen and b) krypton.

The N TALIF scheme used for these experiments is shown in figure 3(a). The twophoton $3 p^{4} S_{3 / 2} \mathrm{~N}$ resonance is accessed via a pair of $206.7 \mathrm{~nm}$ photons and subsequently fluoresces to three different fine structure levels, emitting light respectively at $742.6 \mathrm{~nm}$, $744.4 \mathrm{~nm}$ and $747.0 \mathrm{~nm}$ [23]. This resulting fluorescence is captured by a Hamamatsu Photo Sensor Module H7422P-50, infra-red sensitive photomultiplier (PMT). Stray light originating primarily from the background plasma emission is rejected both spectrally and spatially. A narrow $10 \mathrm{~nm}$ bandpass filter centered at $750 \mathrm{~nm}$ (Thorlabs FB750-10), blocks a significant amount of the emission from the plasma as well as scattering from the UV probe laser. However, there still exists a considerable amount of plasma emission originating from the $\mathrm{N}_{2}$ first positive system near $750 \mathrm{~nm}$ which is well-overlapped with the resulting laser fluorescence. A $300 \mu \mathrm{m}$ pinhole is used to reduce this contribution based on the expectation that the plasma emission originates from a comparatively larger volume than the fluorescence. The pinhole therefore spatially filters out more of the plasma emission than the fluorescence signal, and increases the signal to noise ratio. Both the bandpass filter and the pinhole are attached to the entrance of the PMT.

As shown in figure 1, the light collection optics additionally comprise a 1 inch diameter, $75 \mathrm{~mm}$ focal length plano-convex lens which collimates the fluorescence, followed by another 1 inch diameter, $100 \mathrm{~mm}$ focal length plano-convex lens responsible for focusing 
the light into the PMT. Both lenses are separated by a UV turning mirror to allow precise alignment of the focused light through the pinhole and onto the PMT. The fluorescence is collected orthogonal to the beam propagation axis, via an aperture located beneath the capillary. A glass window attached to this aperture also serves to reject any stray UV light scattered off the quartz tube.

To obtain quantitative atomic nitrogen number densities, signal calibration is performed against a noble gas, krypton $(\mathrm{Kr})$, which has a spectrally close two-photon resonance at $204.1 \mathrm{~nm}$. The idea is based on using the fluorescence from a gas of known number density $(\mathrm{Kr})$, to calibrate the fluorescence measured from another gas $(\mathrm{N})$, so as to infer the number density of this second gas. In most instances, this other gas is typically an unstable atomic species, in which production of controlled or known amounts (viz. number densities) is challenging. This approach of inert gas calibration was proposed in seminal work by [24] and has since been widely adopted in the literature. One advantage of this method is that any uncertainty in the $\mathrm{N}$ signal due to the laser intensity variation along the beam propagation axis - bearing in mind that the probe volume is not located exactly at the beam waist - is minimized since the same intensity variation is present when performing the $\mathrm{Kr}$ calibration. It is therefore imperative that an identical setup is used (with respect to the two-photon pumping and the fluorescence collection) for both the $\mathrm{N}$ and $\mathrm{Kr}$ measurements. Even then however, it is possible that some systematic error could still be present. This is addressed in greater detail in section 3.4.

The $\mathrm{Kr}$ TALIF scheme used is shown in figure 3(b). Absorption of two $204.1 \mathrm{~nm}$ photons from the $4 p^{6}{ }^{1}$ So ground state of $\mathrm{Kr}$ excites electrons to the $5 p^{\prime}[3 / 2]^{2}$ resonance. This population subsequently relaxes to the $5 s^{\prime}[1 / 2]^{1}$ state accompanied by the emission of $826 \mathrm{~nm}$ light. Given that only the $826 \mathrm{~nm}$ fluorescence from the $5 p^{\prime}[3 / 2] 2$ excited state is monitored, the optical branching ratio $\left(\frac{a_{K r}}{A_{K r}}\right)$ is thus less than unity, and is assigned a value of 0.962 based on an average of the values in $[23,24]$. To isolate the atomic fluorescence, a $10 \mathrm{~nm}$ bandpass filter centered at $830 \mathrm{~nm}$ is used. As evident from equation (2) below, while the choice of $\mathrm{Kr}$ as a calibration gas is advantageous since it has a two-photon resonance close to that of $\mathrm{N}$, it is not a perfect candidate since the $\mathrm{Kr}$ fluorescence does not exhibit similarly good overlap. Transmission efficiencies of the various components within the light collection system at these different fluorescent wavelengths must therefore be accounted for.

Following [24], the time and spectrally integrated fluorescence $S$ of two gases - in this case $\mathrm{N}$ and $\mathrm{Kr}$ - may be written respectively as:

$$
\begin{gathered}
S_{N}=\Gamma_{N} \cdot n_{N} \cdot \sigma_{N} \cdot\left(\frac{I_{L}}{h v}\right)_{N}^{2} \cdot\left(\frac{A_{N}}{A_{N}+Q_{N}}\right) \cdot F_{N} \cdot(P M T)_{N} \\
S_{K r}=\Gamma_{K r} \cdot n_{K r} \cdot \sigma_{K r} \cdot\left(\frac{I_{L}}{h v}\right)_{K r}^{2} \cdot\left(\frac{a_{K r}}{A_{K r}}\right) \cdot\left(\frac{A_{K r}}{A_{K r}+Q_{K r}}\right) \cdot F_{K r} \cdot(P M T)_{K r} .
\end{gathered}
$$


Here $\Gamma$ represents the photon statistical factor at a relevant laser wavelength, $n$ is the number density, $\sigma$ is the two-photon absorption cross-section, $I_{L}$ is the laser intensity, $h$ is the Planck constant, $v$ is the laser frequency, $a$ is the natural radiative rate for a specific observed fluorescence transition, $A$ and $Q$ are the natural radiative and quenching rates of the two-photon excited state while $F$ and (PMT) represent the transmission efficiencies of the filters and photomultiplier at the respective fluorescent wavelengths. Rearranging terms in (1.1) and (1.2), one arrives at the following expression for $n_{N}$ the number density of $\mathrm{N}$-atoms:

$$
n_{N}=\left(\frac{\sigma_{K r}}{\sigma_{N}}\right) \cdot\left(\frac{v_{N}}{v_{K r}}\right)^{2} \cdot\left(\frac{P M T_{K r}}{P M T_{N}}\right) \cdot\left(\frac{a_{K r}}{A_{K r}}\right) \cdot\left(\frac{A_{K r}}{A_{N}}\right) \cdot\left(\frac{F_{K r}}{F_{N}}\right) \cdot\left(\frac{A_{N}+Q_{N}}{A_{K r}+Q_{K r}}\right) \cdot\left(\frac{I_{K r}}{I_{N}}\right)^{2} \cdot\left(\frac{S_{N}}{S_{K r}}\right) \cdot n_{K r} .
$$

Given that both the $\mathrm{N}$ and $\mathrm{Kr}$ resonances lie within close spectral proximity, it is assumed that their respective photon statistical factors are invariant within this wavelength range and therefore cancel out in (2). The various parameters on the right hand side of (2) have been grouped as follows: terms in the first four parentheses represents quantities or ratios which have not been measured in this study but referenced from the literature or operating manuals, the next three parentheses contain parameters which have been measured in this work but are also available in the literature, while the remaining terms in (2) consist of only measured quantities.

Due to observed shot-to-shot variations in the laser energy, only laser pulses falling within $\pm 3 \%$ of a predetermined mean energy value (based on the integrated photodiode signal) are accepted for analysis. TALIF PMT signals which satisfy this criterion, are acquired and averaged over a minimum of 300 laser shots. This procedure is observed for all spectral scans, where each scan is conducted with a resolution of about $0.3 \mathrm{pm}$.

A potential source of error not examined in this study is related to observed fluctuations in the temporal profile of the instantaneous laser intensity. The origin of these fluctuations is not well understood but could be due to a slight spatial ellipticity in the unfocused beam profile as visualized using alignment burn paper. This elliptical ratio is roughly 1.8:1 and may cause 'mode-beating' which could lead to irregular spikes in the laser beam intensity with time.

The time evolution of the $\mathrm{N}$-atom density produced by the plasma is measured by varying the time delay between the probe laser pulse and the initiation of the discharge. This is implemented through the use of two BNC 575 delay generators. While in theory a single delay generator would be sufficient for this purpose, a several- $\mu$ s jitter between an input trigger to the HVG until the firing of a high voltage pulse necessitates the use of a second such device. The first delay generator triggers the flashlamp of the Nd:YAG laser followed by the HVG; the trigger input to the HVG produces a synchronization output which is used to trigger the other delay generator. This second delay generator triggers the laser Q-switch after a prescribed time delay, thereby providing a robust scheme for 
varying the time between the arrival of the laser pulse and the firing of the discharge with a jitter of less than $1 \mathrm{~ns}$.

\section{Results and discussion}

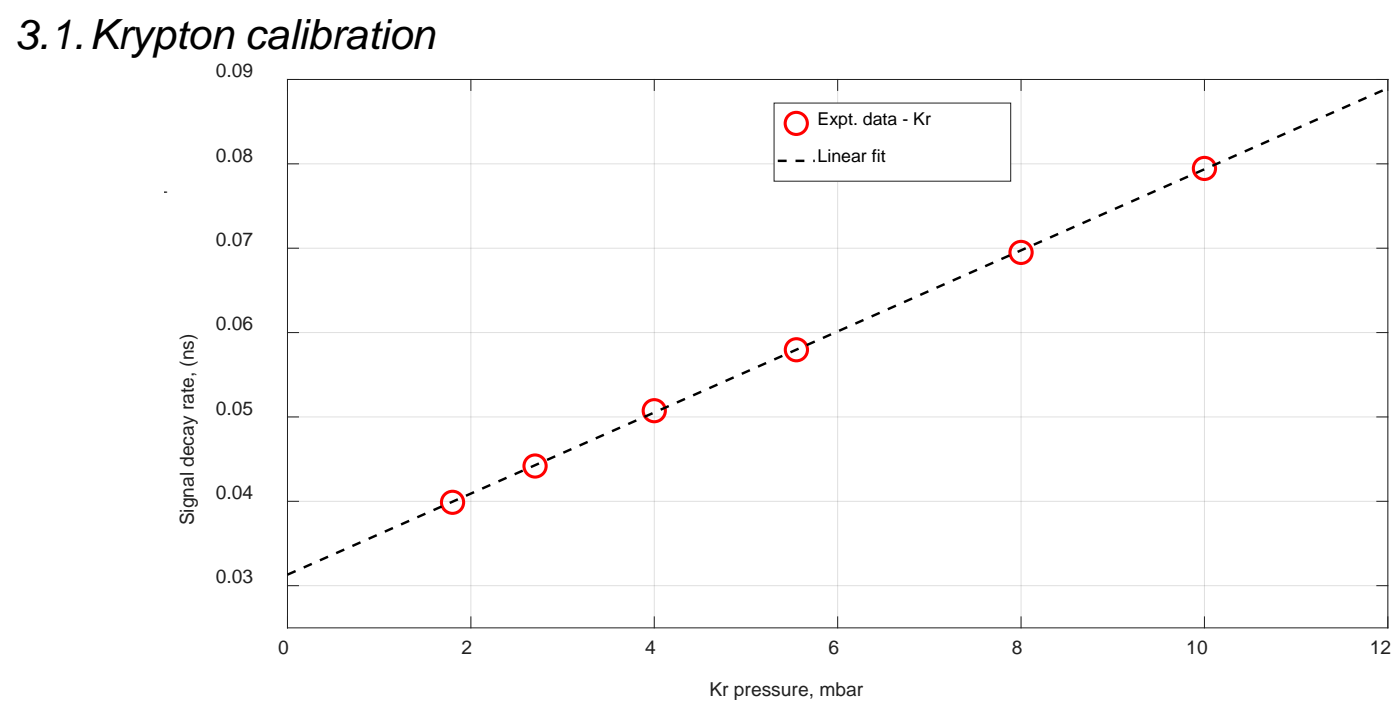

Figure 4. Plot of $\mathrm{Kr}$ TALIF signal decay rate versus $\mathrm{Kr}$ pressure. The random uncertainty of these measurements is about $3 \%$.

Figure 4 shows the Kr TALIF decay rates measured as a function of $\mathrm{Kr}$ pressure. The $y$-intercept of a linear fit to this data gives the natural radiative rate while the gradient of this line provides a measure of the $\mathrm{Kr}$ self-quenching rate. A value of $32.5 \mathrm{~ns}$ (versus 34.1 $\mathrm{ns})$ is obtained for the natural radiative lifetime $\left(A_{K r}\right)^{-1}$ of the $\mathrm{Kr} 5 \mathrm{p}^{\prime}[3 / 2]^{2}$ state, exhibiting excellent agreement with [24]. However, our measured quenching rate $Q_{K r}$ of $1.9 \cdot 10^{-10}$ $\mathrm{cm}^{-3} \mathrm{~s}^{-1}$ (versus $1.46 \cdot 10^{-10} \mathrm{~cm}^{-3} \mathrm{~s}^{-1}$ ) only offers relatively modest agreement [24]. The experimentally measured values of the corresponding natural radiative rate $A_{K r}$ and decay rate $\left(A_{K r}+Q_{K r}\right)$ have been used for computing the absolute $\mathrm{N}$-atom density based on (2), using a reference $\mathrm{Kr}$ pressure of $2.7 \mathrm{mbar}$. The overall effect of the systematic uncertainty or difference between the present measurements and those in [24] on the N-atom density measurements based on equation (2) is calculated to be $<7 \%$.

\subsection{Fluorescence spectra}

Both the fluorescence spectrum of $\mathrm{N}$ and $\mathrm{Kr}$ are shown in figure 5. The purpose of acquiring these spectra are twofold: to compare the measured line intensities particularly for $\mathrm{N}$ which comprises 3 lower energy levels - with that in the literature, and to compare the measured transmission of the $\mathrm{N}$ and $\mathrm{Kr}$ bandpass filters with the numbers in their respective datasheets. The latter is accomplished by performing a separate set of 
experiments, and attaching each of the bandpass filters before the entrance of the spectrometer. The fluorescence spectra is acquired using an ANDOR SR-500i-B1 infrared sensitive spectrometer and averaged over 1000 laser shots. Figure 5(a) shows that the three fine structure $\mathrm{N}$-atom lines are faithfully captured in the experimental emission spectrum. The relative individual linestrengths, computed by taking the spectral integral of each line and normalizing it by the total spectral integral, exhibit excellent agreement with NIST published data [23] as shown in the inset of figure 5(b).

As expected, the $\mathrm{Kr}$ spectrum shows the presence of the $826 \mathrm{~nm}$ atomic fluorescence. However, an exception is that at higher pressures in excess of $9 \mathrm{mbar}$, the presence of two other lines at $828 \mathrm{~nm}$ and $830 \mathrm{~nm}$ is observed. The source of these extraneous emission lines has not been further examined, but could be associated with either collisional processes or electron-ion recombination into various excited states. It is important to point out that these lines fall within the transmission linewidth of commonly used bandpass filters for the $\mathrm{Kr} 5 p^{\prime}[3 / 2]^{2}-5 s^{\prime}[1 / 2]^{1}$ transition, and may constitute a source of error when interpreting PMT data, if not taken into consideration. Interestingly, the presence of additional fluorescence features has also been observed in femtosecondTALIF related research $[25,26]$.

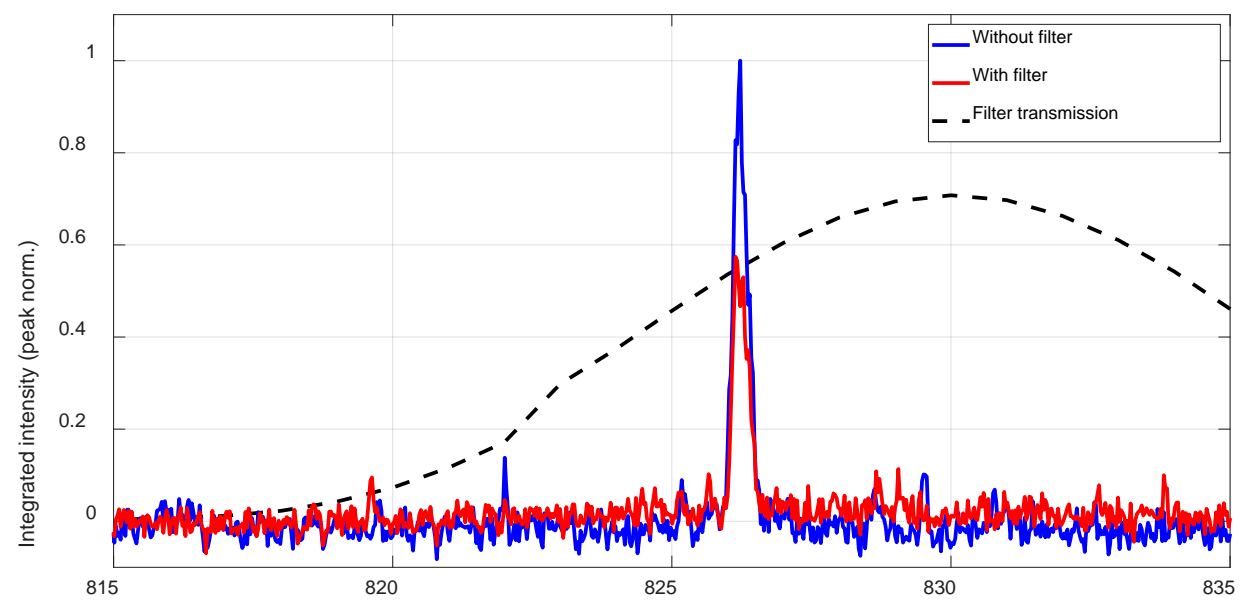

(a)

Wavelength, nm 


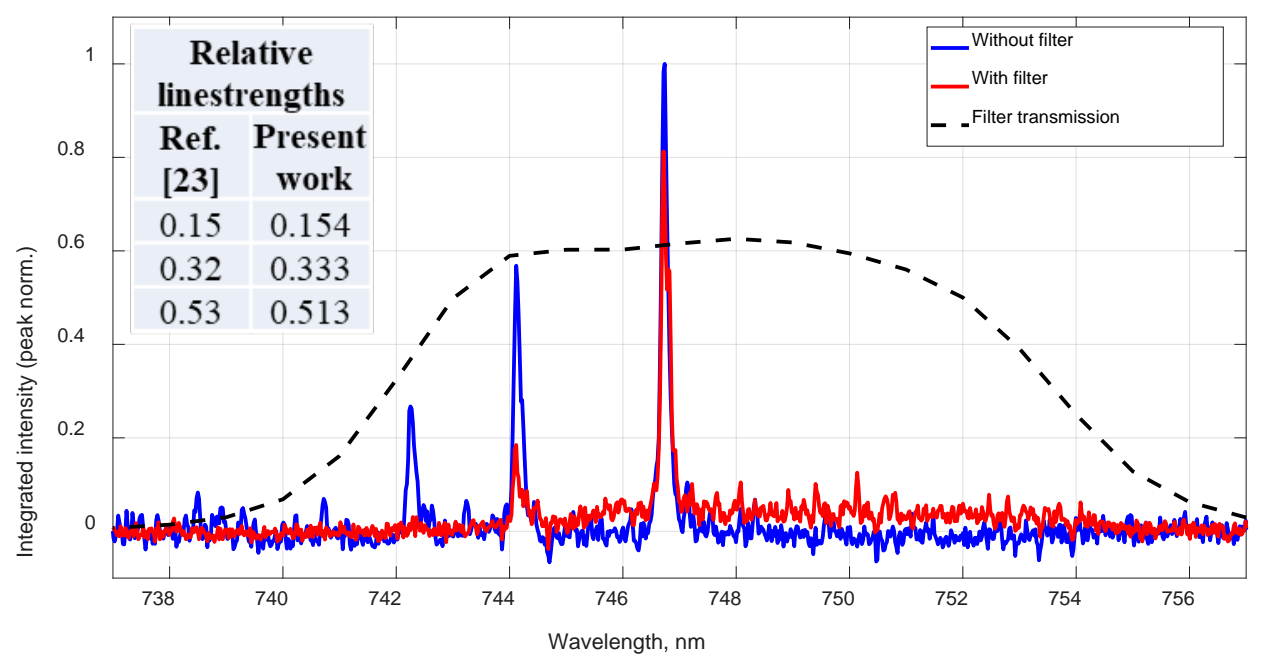

(b)

Figure 5. Peak normalized emission spectra for a) $\mathrm{Kr}$ at 2.7 mbar and b) $\mathrm{N}$. The $\mathrm{N}$ atom spectra are acquired at $\mathrm{t} \approx 160 \mu$ s after discharge initiation.

An evaluation of the filtered emission spectra with their respective transmission curves shows very good agreement for the bandpass filter used for $\mathrm{Kr}$ but only fair agreement for that of N. Fortuitously, the effective transmission - given by the product of the respective normalized linestrengths with either the experimental or datasheet values only differ by around $1 \%$, and no further attempt is made to understand this discrepancy.

\subsection{TALIF signal versus laser intensity}

With reference to equations (1), a linear relationship between the measured fluorescence (viz. TALIF signal) and number density of the species of interest is valid only if the signal observes a quadratic dependence with the laser intensity. Satisfying this condition, also commonly known as the 'linear regime', is typically taken as an indication - based on a classical rate equation analysis - that additional processes such as stimulated emission, photoionization and photodissociation are less important and may be neglected compared to the fluorescence [24]. 


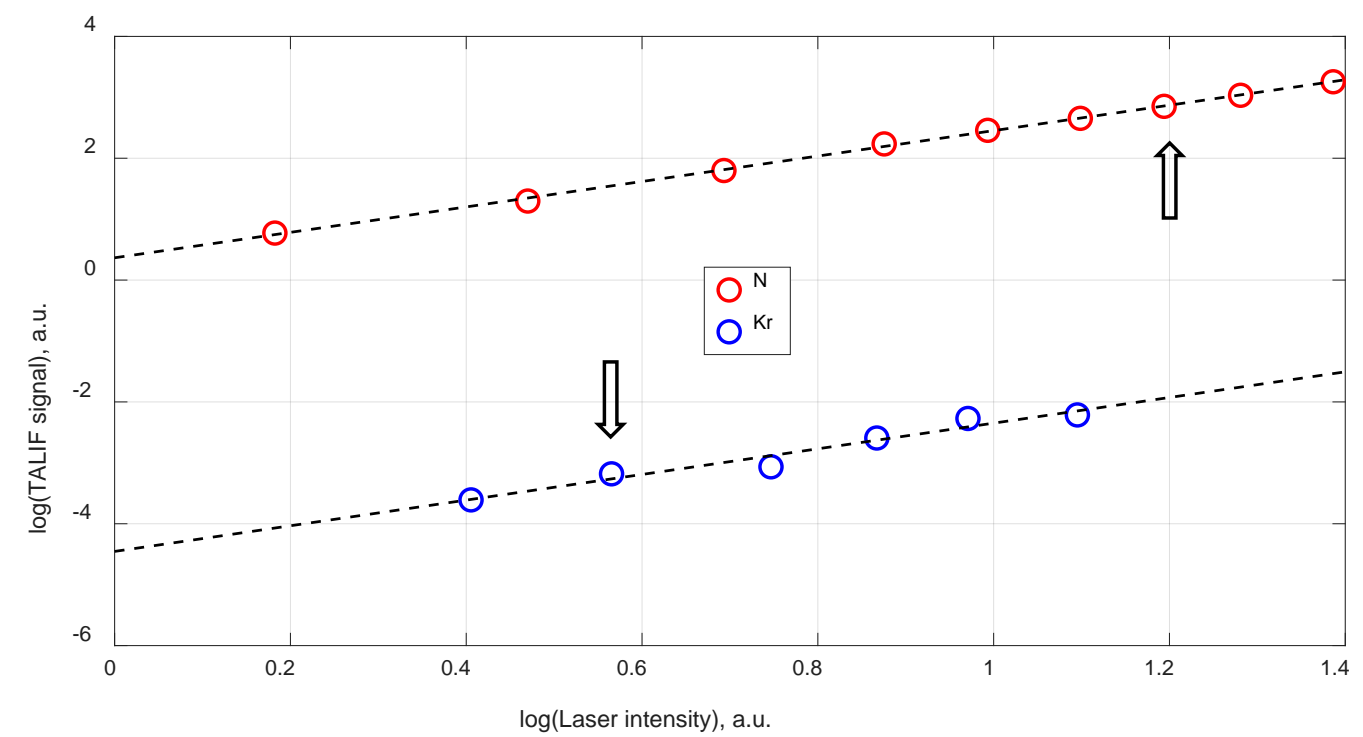

Figure 6. Logarithmic plots of TALIF signals versus laser intensity for $\mathrm{Kr}$ and $\mathrm{N}$. The laser intensities used in the respective experiments are indicated with black arrows.

To ensure operation within this 'linear regime', TALIF signals at resonance are measured for various levels of the laser energy. Plotting the logarithmic values of both the TALIF signal and the laser intensity (given by the integral of the photodiode signal) as displayed in figure 6, yields a slope of about 2 for both the case of $\mathrm{Kr}(2.11)$ and $N(2.08)$. Although full spectral scans are not taken for these data, a similar procedure whereby fluctuations in the laser intensity are limited to $\pm 3 \%$ of a mean value is still observed. For the primary experiments described in section 3.4, the laser energy used in the case of $\mathrm{N}$ was about $85 \mu \mathrm{J}$ and the corresponding value for $\mathrm{Kr}$ was about $30 \mu \mathrm{J}$. Since the measurement volume is not located at the beam focus, we have chosen to express the laser energy in arbitrary units to prevent misinterpreted estimates of the actual laser intensity. As a final note, since the TALIF signals are also dependent on the square of the temporal variation in the laser intensity, the data is also corrected for small differences in the mean temporal profile of the laser pulse for $\mathrm{Kr}$ and $\mathrm{N}$. These small differences are believed to arise from the non-uniform lasing efficiency of the dye at the slightly different resonant wavelengths. 


\subsection{Time evolution of $\mathrm{N}$-atom density}

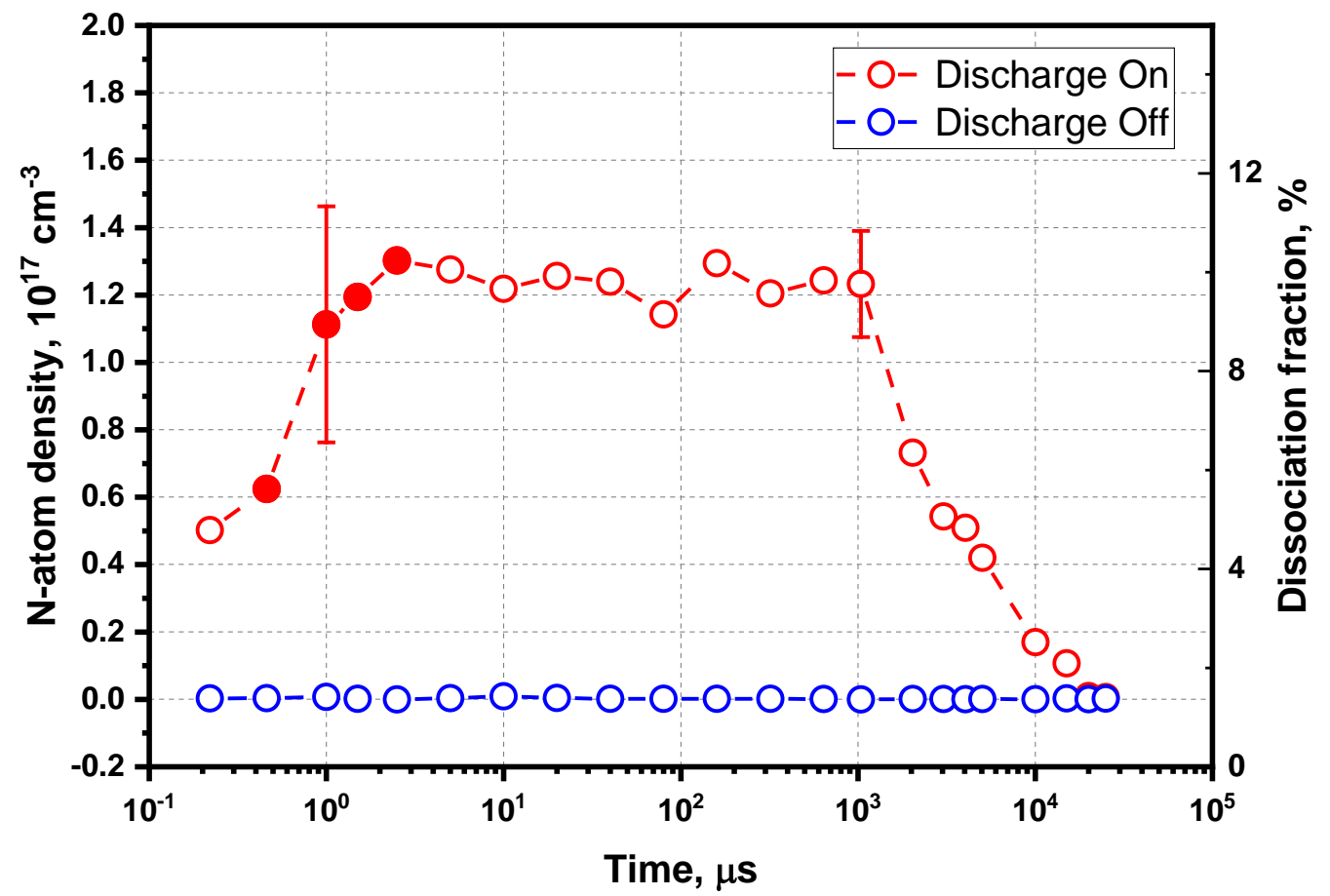

Figure 7. Time evolution of the $\mathrm{N}$-atom density measured using TALIF. Red filled symbols represent data points where quenching corrections have not been accurately implemented.

With the benefit of the data presented in the foregoing sections, the time evolution of the $\mathrm{N}$-atom density produced by the discharge is calculated based on equation (2) and shown in figure 7 . The primary source of systematic error in these measurements stems from the difference in radial profiles for the $\mathrm{N}$ and $\mathrm{Kr}$ experiments. Recall that since the capillary is located some distance upstream of the focus of the probe laser, this means that the exact measurement volume of the TALIF signal is not clearly defined. In particular, it is quite likely that the signal originates from a finite radial extent of the capillary cross-section, rather than just the center. While replicating the same configuration in $\mathrm{Kr}$ partially mitigates this uncertainty, it is believed that the difference in radial profiles of the $\mathrm{N}$ and $\mathrm{Kr}$ atoms within the capillary will constitute a certain amount of error. In particular, a previous study in the same discharge noted a ' $U$ ' shape distribution of $\mathrm{N}_{2}\left(\mathrm{C}^{3} \Pi_{\mathrm{u}}, \mathrm{v}=0\right)$ molecules over the capillary cross-section during the discharge pulse [20]. The consequent $\mathrm{N}$-atom production is expected to be strongly influenced by and replicate this initial distribution. In contrast, the $\mathrm{Kr}$ atoms are likely to be more uniformly distributed over the entire section of the capillary. Other sources of error include the imperfect shot to shot repeatability of the discharge, as well as the uncertainty in the ratio of the $\mathrm{N}$ and $\mathrm{Kr}$ two-photon cross-sections. The error bars in figure 
7 have been obtained as a result of repeated experiments at two different collection angles and at reduced laser energies.

The $\mathrm{N}$-atom density is tracked from early times - about $300 \mathrm{~ns}$ after the first HV pulse - until several tens of milliseconds after the initiation of the discharge. As seen in figure 8 , there is considerable emission from the plasma which spectrally overlaps with the fluorescence. This emission, which is observed together with each incident HV pulse, lasts for about $1 \mu \mathrm{s}$ and can potentially overwhelm the TALIF signal if not adequately addressed. To improve the signal to noise ratio, measurements for $t<1 \mu \mathrm{s}$ are timed such that they correspond to the decaying section of the plasma emission. At the other end of the time spectrum, care is also taken to ensure that measurements are conducted well in advance of the gas renewal time $t_{r}=40 \mathrm{~ms}$ and the laser pulse repetition time (i.e. $1 / 10 \mathrm{~Hz}=100 \mathrm{~ms})$.

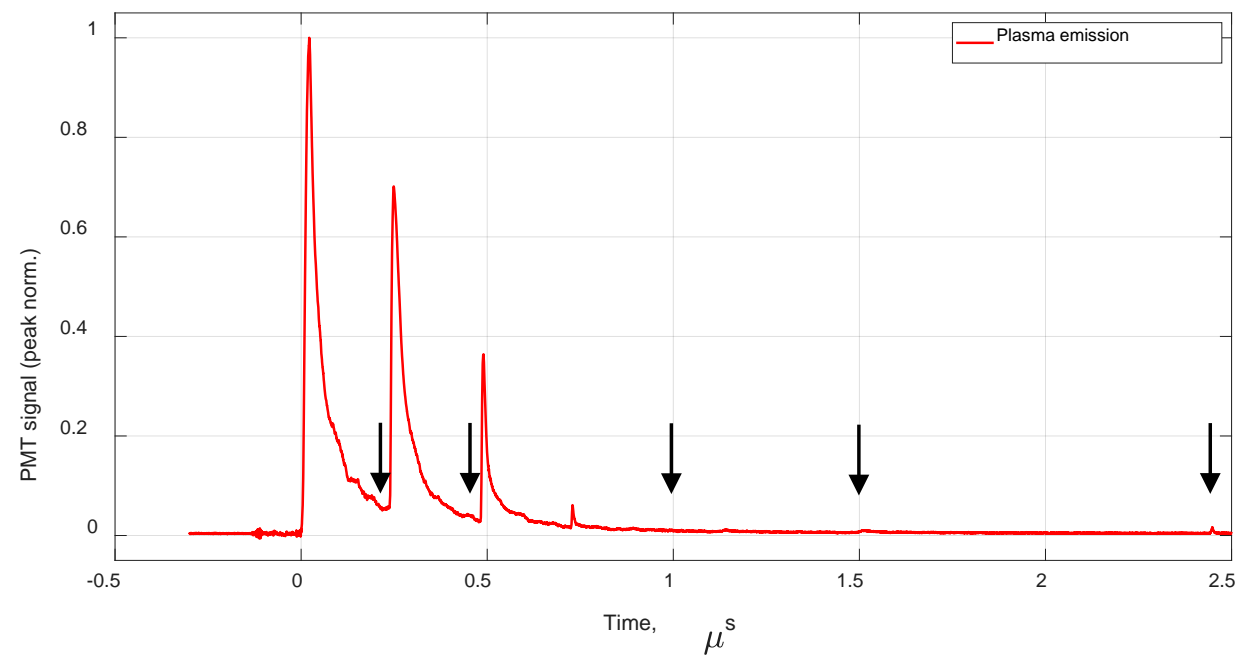

Figure 8. PMT time trace acquired during the early afterglow of the discharge, illustrating the strong background emission from the plasma up to about $t=1 \mu \mathrm{s}$. (Note that the small peak near $t=2.5 \mu$ s provides an example of a representative TALIF signal.) Black arrows correspond to time instants of the TALIF measurements relative to this emission signal.

As described earlier in section 2.2, the TALIF signals $S_{N}$ and $S_{K r}$ used to compute the $\mathrm{N}$ number densities for each data point in figure 7 corresponds to a spectral scan over the atomic line taken at that particular time instant. For each scan, in addition to any nonresonant plasma emission, a background component anticipated to be originating from the fluorescence of the quartz capillary, is subtracted from the measured TALIF signals. This contribution persists even several hundred picometers away from the peak signal wavelength, and is determined by simply tuning the probe laser off-resonance (on the basis that its value is independent of wavelength within this range). Such interference 
from quartz fluorescence has also been observed and characterized in previous work [18]. Furthermore, to eliminate the possibility of $\mathrm{N}$-atom production by laser photolysis or outside of the capillary, it is confirmed (see figure 7) that no TALIF signals are registered in the absence of the discharge. Finally, wherever possible, quenching corrections are applied based on experimentally determined TALIF decay rates rather than rates published in the literature. This approach is deemed prudent given uncertainties surrounding the exact composition of the plasma with time (from the initiation of the discharge). Unfortunately, due to the existence of fast quenching processes, not all the data could be corrected. This issue is addressed in greater detail in section 3.5.

\subsection{Time evolution of $\mathrm{N}$-atom TALIF fluorescence decay rates}

Figure 9 echoes the time evolution plot of figure 7 but instead displays the experimentally measured TALIF signal decay rates. It is observed that at early times, mainly for $t<5 \mu \mathrm{s}$, the measured decay rates are not resolvable $\left(\frac{1}{\tau_{\text {decay }}}=\left(A_{N}+Q_{N}\right) \gtrsim\right.$ $0.4 \mathrm{~ns}^{-1}$ ), and are limited by the duration of the probe laser pulse. For these data points, the $\mathrm{N}$-atom density has been computed by using the measured signal decay rates, which obviously will lead to an underestimation of the actual values.

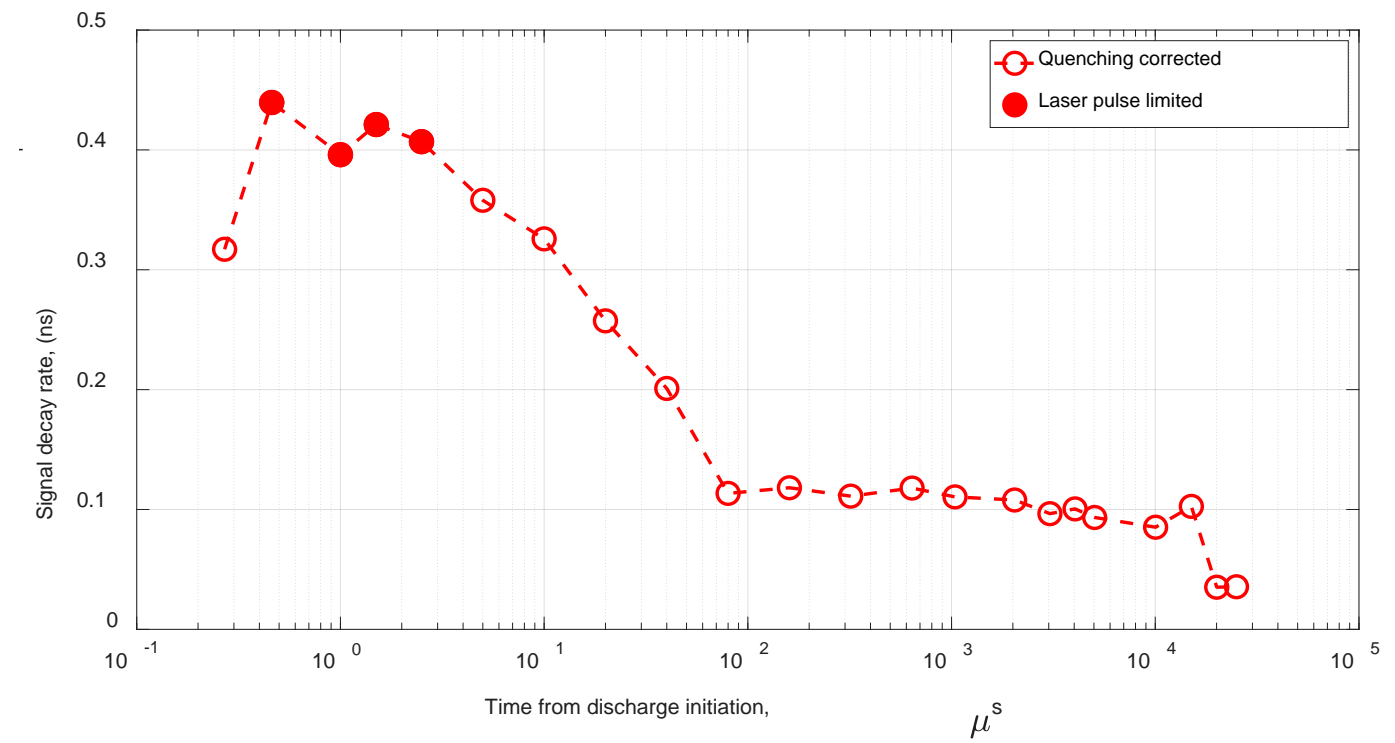

Figure 9. Time evolution of the $\mathrm{N}$-atom TALIF signal decay rate. The maximum random uncertainty of these measurements is about $5 \%$ and $15 \%$ for $t \leq 10 \mathrm{~ms}$ and $t>10 \mathrm{~ms}$ respectively.

The existence of these rapid decay rates merits further discussion, especially with regards to the origin of the underlying processes. Electron production and its ensuing impact on the plasma kinetics is a distinctive feature of such capillary discharges; estimates of the electron density are about $10^{14} \mathrm{~cm}^{-3}$, about $700 \mathrm{~ns}$ after the initiation of 
the discharge [27]. Given that electrons are known to be very effective 'quenchers' of excited states under our experimental conditions [27], and that the peak of the electron density tends to coincide with time instants where the TALIF decay rates are fastest, suggests that electron quenching could be a reason for this rapid signal decay. Another possible reason is that the quenching rate of the $\mathrm{N}_{2}$ bath gas could be significantly higher due to an increased gas temperature at these early times ( $t<5 \mu \mathrm{s})$, even though the gas density remains constant (see further discussion in section 4.1). An earlier study conducted under identical conditions measured a temperature rise to about $2150 \mathrm{~K}$ at $t=$ $1.5 \mu \mathrm{s}$ [24]. The temperature after the first HV pulse $(t \approx 270 \mathrm{~ns})$ is however, significantly lower $(\approx 750 \mathrm{~K})$ [24], and could explain why the TALIF decay rate measured at this time (first point in figure 9 ) is less rapid. Finally, as a consequence of the large $\mathrm{N}$-atom densities (on the order of $10^{17} \mathrm{~cm}^{-3}$ ) produced by the plasma, the presence of selfquenching from $\mathrm{N}$-atoms could also be a factor in accelerating the fluorescence decay. Evidence in favor of this argument can also be seen later, for instance at $t=1 \mathrm{~ms}$, when the gas temperature has probably equilibrated with the ambient but with the $\mathrm{N}$-atom density still reasonably high. The measured decay rate at $t=10 \mathrm{~ms}\left(\frac{1}{\tau_{\text {decay }}} \approx 0.1 \mathrm{~ns}^{-1}\right)$ is still slightly higher than that predicted by considering the natural radiative rate and $\mathrm{N}_{2}$ quenching at room temperature $\left(0.06 \lesssim \frac{1}{\tau_{\text {decay }}} \lesssim 0.08 \mathrm{~ns}^{-1}\right)$. The latter is computed based on published room temperature quenching rate coefficients for $\mathrm{N}_{2}[24,28]$.

\section{Discussion}

\subsection{Time evolution of $\mathrm{N}$-atom density}

This section analyzes the main processes responsible for the production and removal of $\mathrm{N}$-atoms in a nanosecond capillary discharge, in light of the fact that the measured peak dissociation fraction of about $9.7 \%$ is noted to be 'unusually' high. The dissociation fraction, $\beta$ is defined as:

$$
\beta=\left(\frac{n_{N}}{n_{N}+2 \cdot n_{N_{2}}}\right)
$$

Returning to figure 7, it is evident that the results display a 'trapezoidal-like' shape a continuous rise in the $\mathrm{N}$-atom density after each of the three HV pulses, lasting up to about $t=2 \mu \mathrm{s}$, followed by a plateau until $t=1 \mathrm{~ms}$, and finally a drop from $2 \mathrm{~ms} \leq t \leq 10$ $\mathrm{ms}$. The initial rise in the number density to about $1.29 \cdot 10^{17} \mathrm{~cm}^{-3}$ is believed to be driven mainly by electron-impact excitation to high lying predissociative states, according to the reaction given in $[29,30]$ : 


$$
e+N_{2} \rightarrow N\left({ }^{4} S\right)+N\left({ }^{2} D\right)
$$

Each HV pulse produces additional $\mathrm{N}$-atoms via reaction (4), of which one atom is in the ground $N\left({ }^{4} S\right)$ state, and the other in the electronically excited state $N\left({ }^{2} \mathrm{D}\right)$. Another source of ground state $\mathrm{N}$-atoms is therefore derived from the collisional de-excitation of these $N\left({ }^{2} \mathrm{D}\right)$ atoms. At gas temperatures between $T=1700-2000 \mathrm{~K}$ typical of such a capillary discharge [20], the rate constant of quenching of $N\left({ }^{2} \mathrm{D}\right)$ atoms

$$
\mathrm{N}\left({ }^{2} \mathrm{D}\right)+\mathrm{N}_{2} \rightarrow \mathrm{N}\left({ }^{4} \mathrm{~S}\right)+\mathrm{N}_{2}
$$

is equal to $k_{5}=(3-4) \cdot 10^{-12} \mathrm{~cm}^{3} / \mathrm{s}$ [31]. A typical quenching time of reaction (5) at $P=$ $27 \mathrm{mbar}$ is equal to $400-500 \mathrm{~ns}$. Thus, at $t>2 \mu \mathrm{s}$, the density of ground state $\mathrm{N}\left({ }^{4} \mathrm{~S}\right)$ atoms is equal to the total density of $\mathrm{N}$-atoms produced in the discharge.

At times beyond $t=2 \mu \mathrm{s}$, the main process influencing the $\mathrm{N}$-atom density is the density change due to gas dynamic rarefaction of the heated region following the initial temperature rise (via fast gas heating). A typical cooling time with the wall based on thermal diffusion is equal to [32]:

$$
v_{h t}{ }^{-1}=\left[\left(\frac{\lambda(T)}{C_{v} \cdot \rho}\right) \cdot\left(\frac{2.4}{R}\right)^{2}\right]^{-1},
$$

where $\lambda(T)$ is the thermal heat conductivity, $C_{v}$ is the isochoric specific heat capacity, and $\rho$ is the gas density. For $T=1000 \mathrm{~K}, R=0.8 \mathrm{~mm}$ and pressure $P=27 \mathrm{mbar}$, this time is equal to $\tau_{h t}=46 \mu \mathrm{s}$. At the same time, for a capillary length, $L \approx 7 \mathrm{~cm}$ and a gas temperature $T \leq 1000 \mathrm{~K}$, a typical travel time of the rarefaction wave to the measurement location (viz. acoustic timescale) is $\tau_{a c} \geq 57 \mu \mathrm{s}$. As a result, the influence of gas hydrodynamics on the $\mathrm{N}$-atom density at the center of the discharge tube, i.e. at equal distances from the electrodes, is minimized. This could explain why the measured $\mathrm{N}$-atom density remains relatively constant at these later times.

In addition, the strong possibility of a non-uniform radial profile of the $\mathrm{N}$-atom density immediately after the discharge [20], suggests that a change in the atom density on the axis of the tube due to diffusion from the near-wall zones is possible. A typical diffusion time can be estimated from [32] as:

$$
v_{\text {diff }}{ }^{-1}=\left[D_{N} \cdot\left(\frac{2.4}{R}\right)^{2}\right]^{-1},
$$

where $D_{N}$ is the mass diffusion coefficient of $\mathrm{N}\left({ }^{4} \mathrm{~S}\right)$ atoms in pure molecular nitrogen, $R$ is the radius of the capillary tube. At $P=27 \mathrm{mbar}, T=2000 \mathrm{~K}, R=0.8 \mathrm{~mm}$ and $D_{N} \approx 150$ $\mathrm{cm}^{2} / \mathrm{s}$ [33], a typical diffusion time is equal to $\tau_{\text {diff }} \leq 10 \mu \mathrm{s}$. The fact that the measured distribution of $\mathrm{N}$-atoms stays almost unchanged from $t=2-10 \mu$ s (see figure 7) may be an indication that the TALIF signals originate from the entire cross-section of the capillary.

A typical time of heterogeneous recombination of $\mathrm{N}\left({ }^{4} \mathrm{~S}\right)$ atoms, $v_{\text {het }}{ }^{-1}$, can be written as [30]: 


$$
v_{\text {het }}{ }^{-1}=\left(\frac{\gamma_{N} \cdot u}{2 R}\right)+\left[D_{N} \cdot\left(\frac{2.4}{R}\right)^{2}\right]^{-1}
$$

where $u$ is the thermal velocity, and $\gamma_{N}$ is a coefficient of heterogeneous recombination of atomic nitrogen on the wall of the capillary tube, $\gamma_{N}=(6-7) \cdot 10^{-4}$ for a clean quartz surface at a wall temperature of between $T_{\text {wall }}=300-600 \mathrm{~K}$ [34]. The recombination coefficient increases with wall temperature, reaching $\gamma_{N}=(1-2) \cdot 10^{-3}$ at $T_{\text {wall }}=800-$ $1200 \mathrm{~K}$ [34]. In the latter case, the time of heterogeneous recombination will be equal to $\tau_{\text {het }}=1.5-3 \mathrm{~ms}$, in reasonable agreement with the results of the current measurements (see figure 7). This indicates that the removal of $\mathrm{N}$-atoms is driven by heterogeneous recombination and that accumulation of $\mathrm{N}$-atoms from pulse to pulse will be absent at repetition frequencies below $100 \mathrm{~Hz}$.

\subsection{Possible mechanisms leading to efficient $N_{2}$ dissociation}

As mentioned earlier, the maximum measured ground state $\mathrm{N}$-atom density is 1.29 . $10^{17} \mathrm{~cm}^{-3}$ and corresponds to a dissociation degree of about $9.7 \%$ based on equation (3). Such high levels of dissociation have rarely been reported in the literature [35], and surpasses typical values measured in streamer discharges [36]. The specific deposited energy over the three HV pulses, averaged over the discharge volume is about $W=1.7$ $\mathrm{eV} /$ molecule. In this case, the fraction of discharge energy spent on dissociation is equal to $50 \%$, corresponding to an energy efficiency of $\mathrm{N}$-atom production (G-factor) of $G_{N}=10$ atom/100 eV (i.e. 10 atoms of nitrogen per $100 \mathrm{eV}$ of deposited energy).

Figure 10 presents the results of measurements [37, 38] and calculations [30] of the energy efficiency of $N_{2}$ dissociation, $G_{N}$, as a function of the reduced electric field E/N. For $E / N=200-250 \mathrm{Td}$, the energy efficiency of $\mathrm{N}\left({ }^{4} S\right)$ production via dissociation of $\mathrm{N}_{2}$ by electron impact (i.e. only reaction (4)) is equal to $G_{N}=1-1.3$ atom/100 eV [30] as shown by the blue curve. This is almost 10 times lower than the value obtained in the present work for the nanosecond capillary discharge, albeit where both the reduced electric field and specific deposited energy are high. Given such a large difference, we therefore attempt to make further sense of the experimental results in the following section.

One of the immediate consequences of having both a high $E / N$ and large specific energy deposited to the discharge, is a high density of electrons (peak $n_{e} \geq 10^{15} \mathrm{~cm}^{-3}$ [27]) and electronically excited $\mathrm{N}_{2}$ molecules. To this end, we consider the dissociation of electronically excited molecules by electron impact, such as:

$$
\mathrm{e}+\mathrm{N}_{2}\left(\mathrm{~A}^{3} \Sigma_{\mathrm{u}}^{+}, \mathrm{B}^{3} \Pi_{\mathrm{g}}, \mathrm{C}^{3} \Pi_{\mathrm{u}}\right) \rightarrow \mathrm{e}+\mathrm{N}\left({ }^{4} \mathrm{~S}\right)+\mathrm{N}\left({ }^{4} \mathrm{~S},{ }^{2} \mathrm{D}\right)
$$




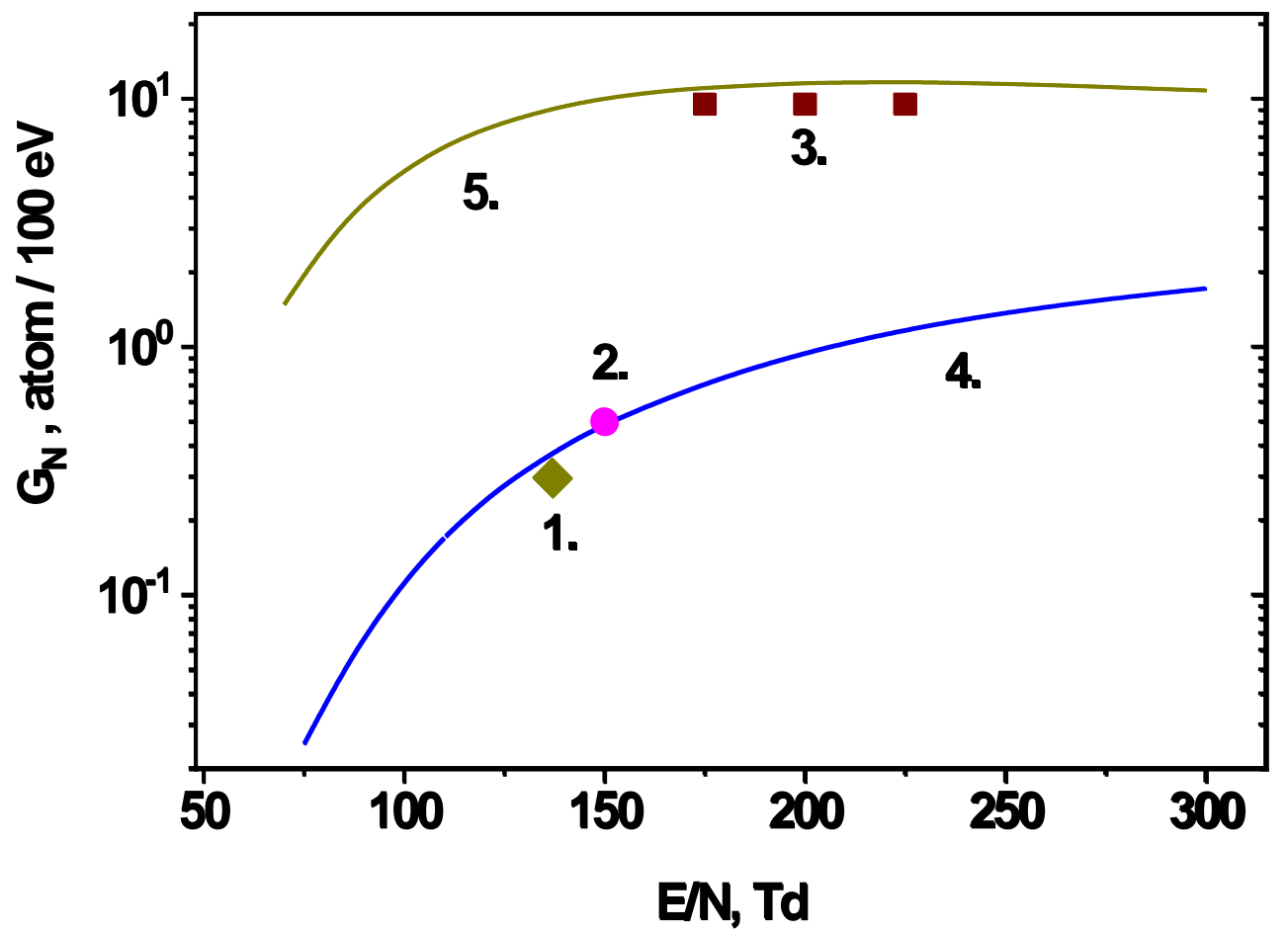

Figure 10. Energy efficiency of dissociation of $\mathrm{N}_{2}\left(\mathrm{G}\right.$-factor, $G_{N}$, is a number of atoms per $100 \mathrm{eV}$ of deposited energy) as a function of $E / N$. Symbols are the results of measurements: 1 - [37], 2 - [38], 3 - the present work; 4 - the results of calculations [30], 5 - calculations of the present work taking into account stepwise electron impact dissociation via $\mathrm{N}_{2}\left(\mathrm{~A}^{3} \Sigma_{\mathrm{u}}^{+}, \mathrm{B}{ }^{3} \Pi_{\mathrm{g}}, \mathrm{C}^{3} \Pi_{\mathrm{u}}\right)$ electronic states.

As will be seen, reaction (9) provides a significant input of $\mathrm{N}$ atoms under the conditions of the present work. Calculated rate constants of the dissociation of $\mathrm{N}_{2}\left(\mathrm{~A}^{3} \Sigma_{\mathrm{u}}^{+}, \mathrm{B}{ }^{3} \Pi_{\mathrm{g}}, \mathrm{C}^{3} \Pi_{\mathrm{u}}\right)$ excited states by electron impact as a function of $E / N$ are presented in figure 11 . The data are obtained by solving the Boltzmann equation in a twoterm approximation, using BOLSIG+ [39]. Self-consistent sets of the cross-sections for elastic and inelastic electron collisions with $\mathrm{N}_{2}$ molecules have been taken from [40], and cross-sections for reaction (9) from [41]. It should be emphasized that for $n_{e} \geq 10^{15} \mathrm{~cm}^{-3}$ and $E / N \geq 75 \mathrm{Td}$ (i.e. typical conditions during the HV pulse), reaction (9) is faster than the relevant depletion processes involving quenching of the $\mathrm{N}_{2}\left(\mathrm{~A}^{3} \Sigma_{\mathrm{u}}^{+}, \mathrm{B}{ }^{3} \Pi_{\mathrm{g}}, \mathrm{C}{ }^{3} \Pi_{\mathrm{u}}\right)$ states to the $\mathrm{N}_{2}\left(\mathrm{X}^{1} \Sigma_{\mathrm{g}}^{+}\right)$ground state, given by:

$$
\begin{gathered}
\mathrm{e}+\mathrm{N}_{2}\left(\mathrm{~A}^{3} \Sigma_{\mathrm{u}}^{+}, \mathrm{B}{ }^{3} \Pi_{\mathrm{g}}, \mathrm{C}{ }^{3} \Pi_{\mathrm{u}}\right) \rightarrow \mathrm{e}+\mathrm{N}_{2}\left(\mathrm{X}^{1} \Sigma_{\mathrm{g}}^{+}\right), \\
\mathrm{N}_{2}\left(\mathrm{~A}^{3} \Sigma_{\mathrm{u}}^{+}, \mathrm{B}{ }^{3} \Pi_{\mathrm{g}}, \mathrm{C}^{3} \Pi_{\mathrm{u}}\right)+\mathrm{N}_{2} \rightarrow \mathrm{N}_{2}\left(\mathrm{X}^{1} \Sigma_{\mathrm{g}}^{+}\right)+\mathrm{N}_{2}, \\
\mathrm{~N}_{2}\left(\mathrm{~A}^{3} \Sigma_{\mathrm{u}}^{+}\right)+\mathrm{N}_{2}\left(\mathrm{~A}^{3} \Sigma_{\mathrm{u}}^{+}\right) \rightarrow \mathrm{N}_{2}\left(\mathrm{~B}^{3} \Pi_{\mathrm{g}}, \mathrm{C}^{3} \Pi_{\mathrm{u}}\right)+\mathrm{N}_{2}\left(\mathrm{X}^{1} \Sigma_{\mathrm{g}}^{+}\right) .
\end{gathered}
$$


As an illustrative comparison, the quenching rate constant of the $\mathrm{N}_{2}\left(\mathrm{~B}^{3} \Pi_{\mathrm{g}}\right)$ state to the $\mathrm{N}_{2}\left(\mathrm{X}^{1} \Sigma_{\mathrm{g}}^{+}\right)$ground state by electron impact is shown in figure 11 . Clearly, for $E / N \geq 75$ $\mathrm{Td}$, this reaction is much slower than the dissociative quenching pathways in reaction (9). A similar argument applies as well for the quenching rate constants of the $\mathrm{N}_{2}\left(\mathrm{~A}^{3} \Sigma_{\mathrm{u}}^{+}\right)$and $\mathrm{N}_{2}\left(\mathrm{C}^{3} \Pi_{\mathrm{u}}\right)$ states. Likewise, at $\mathrm{ne}_{\mathrm{e}}>10^{15} \mathrm{~cm}^{-3}$, the frequency of dissociative quenching in the discharge exceeds $2 \cdot 10^{7} \mathrm{~s}^{-1}$, which is $5-6$ times higher than the frequency of $\mathrm{N}_{2}\left(\mathrm{~A}^{3} \Sigma_{\mathrm{u}}^{+}\right)$quenching in the pooling reaction. Thus, at $E / N>75 \mathrm{Td}$ the total rate of dissociation given by reaction (9) is equal to the total rate of excitation of these states by electron impact. It should be added that while fast transitions between the $\mathrm{N}_{2}\left(\mathrm{~A}^{3} \Sigma_{\mathrm{u}}^{+}, \mathrm{B}{ }^{3} \Pi_{\mathrm{g}}, \mathrm{C}{ }^{3} \Pi_{\mathrm{u}}\right)$ triplet states through collisions with electrons are possible [27], this exchange does not change the gross population of these 3 triplet states.

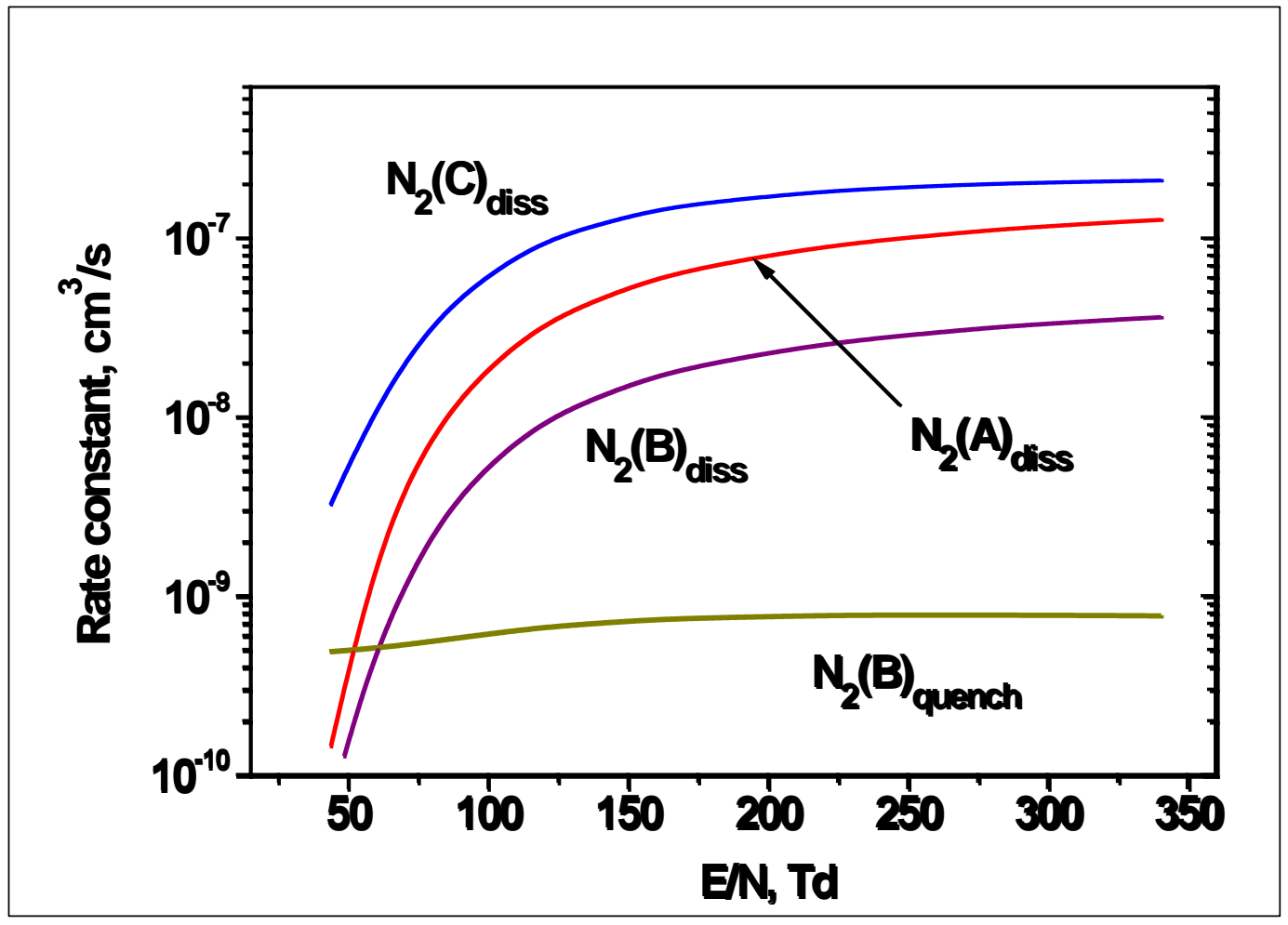

Figure 11. Rate constants of dissociation of $\mathrm{N}_{2}\left(\mathrm{~A}^{3} \Sigma_{\mathrm{u}}^{+}, \mathrm{B}{ }^{3} \Pi_{\mathrm{g}}, \mathrm{C}^{3} \Pi_{\mathrm{u}}\right)$ by electron impact and for the quenching of $\mathrm{N}_{2}\left(\mathrm{~B}^{3} \Pi_{\mathrm{g}}\right)$ to the $\mathrm{N}_{2}\left(\mathrm{X}^{1} \Sigma_{\mathrm{g}}^{+}\right)$ground state by electron impact as a function of $E / N$. The data are calculated on the basis of cross-sections obtained from [41]. 
Figure 12 compares the calculated and measured excitation coefficients $K_{e x} / V_{d r}$ for the $\mathrm{N}_{2}\left(\mathrm{~A}^{3} \Sigma_{\mathrm{u}}^{+}, \mathrm{B}{ }^{3} \Pi_{\mathrm{g}}, \mathrm{C}{ }^{3} \Pi_{\mathrm{u}}\right)$ excited states, where $K_{e x}=k_{A}+k_{B}+k_{C}$, and $V_{d r}$ is the drift velocity of electrons for a given $E / N$. The measured excitation coefficients [42] are shown as points and are assumed to include the contributions of cascading from all of the triplet states $\mathrm{N}_{2}\left(\mathrm{~A}^{3} \Sigma_{\mathrm{u}}^{+}, \mathrm{B}^{3} \Pi_{\mathrm{g}}, \mathrm{C}^{3} \Pi_{\mathrm{u}}\right)$. These data are in turn used to estimate the energy efficiency of $\mathrm{N}_{2}$ dissociation, given by:

$$
G_{N}=\frac{2 \cdot\left(k_{A}+k_{B}+k_{C}\right)}{V_{d r} \cdot E / N} .
$$

The results of calculations according to formula (11) are given in figure 10 (curve 5). Comparison of these numerical results with the experimental data prove that the energy efficiency of $\mathrm{N}_{2}$ dissociation in the nanosecond capillary discharge is close to the maximum possible value. In particular, the $5-10$ times larger production of $\mathrm{N}_{2}\left(\mathrm{~A}^{3} \Sigma_{\mathrm{u}}^{+}, \mathrm{B}{ }^{3} \Pi_{\mathrm{g}}, \mathrm{C}^{3} \Pi_{\mathrm{u}}\right)$ molecules (due to the high reduced fields and electron densities associated with the capillary discharge) compared with conventional electron impact dissociation of ground state molecules given in reaction (4), implies that the corresponding $\mathrm{N}$-atom production is also $5-10$ times more efficient.

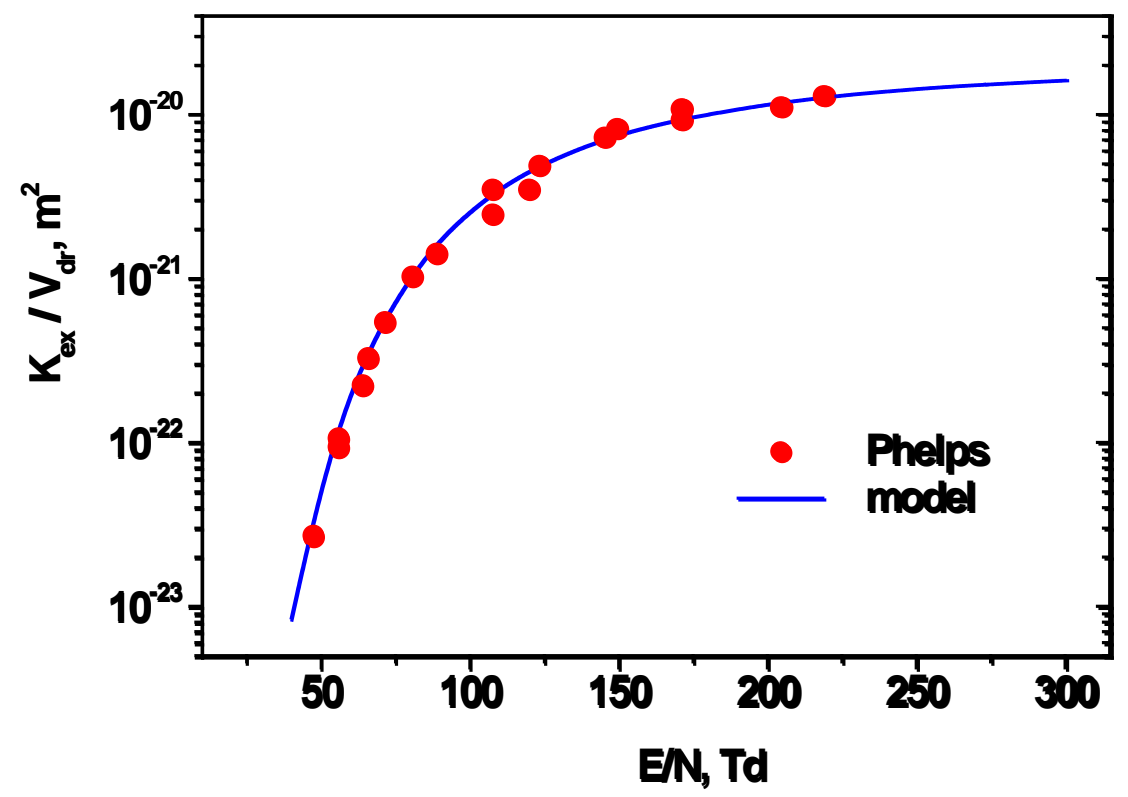

Figure 12. Effective coefficient of electron impact excitation of the triplet states of nitrogen $\mathrm{N}_{2}\left(\mathrm{~A}^{3} \Sigma_{\mathrm{u}}^{+}, \mathrm{B}{ }^{3} \Pi_{\mathrm{g}}, \mathrm{C}{ }^{3} \Pi_{\mathrm{u}}\right)$ as a function of $E / N$. Symbols are for the experimental data [42], the line is the result of the calculation of the present work.

Summarizing, the key to maximizing the dissociation efficiency as experimentally observed in the present work, is as follows: (i) the ionization degree should be high 
enough to ensure that reaction (9) is the main pathway for the dissociative quenching of nitrogen triplet states $\mathrm{N}_{2}\left(\mathrm{~A}^{3} \Sigma_{\mathrm{u}}^{+}, \mathrm{B}{ }^{3} \Pi_{\mathrm{g}}, \mathrm{C}{ }^{3} \Pi_{\mathrm{u}}\right)$; (ii) the duration of the discharge pulse is much longer than the characteristic dissociation time due to reaction (9) so that the electrons are maintained at sufficiently high energies.

\section{Conclusions}

$\mathrm{N}$-atom density measurements have been conducted in a pure $\mathrm{N}_{2}$ nanosecond pulse capillary discharge at a pressure of 27 mbar. This work forms part of a larger effort to understand the kinetics of discharges with both high levels of specific deposited energy and reduced electric fields. A peak $\mathrm{N}$-atom density of about $1.29 \cdot 10^{17} \mathrm{~cm}^{-3}$, corresponding to an exceptionally high dissociation degree of about $9.7 \%$, has been measured using two-photon absorption laser induced fluorescence. The energy efficiency, $G_{N}$, of the $\mathrm{N}$-atom production, reaches approximately 10 atoms $/ 100 \mathrm{eV}$, and suggests the existence of alternative pathways for the generation of these atomic species. In order to justify these experimental observations, we propose a stepwise dissociation mechanism via electronically excited $\mathrm{N}_{2}\left(\mathrm{~A}^{3} \Sigma_{\mathrm{u}}^{+}, \mathrm{B}{ }^{3} \Pi_{\mathrm{g}}, \mathrm{C}^{3} \Pi_{\mathrm{u}}\right)$ triplet states, to complement the more well-known pathway of electron impact dissociation of $\mathrm{N}_{2}\left(\mathrm{X}^{1} \Sigma_{\mathrm{g}}^{+}\right)$ ground state molecules. The present study supports the development of the capillary discharge as an efficient source of $\mathrm{N}$-atoms.

\section{Acknowledgments}

This work was partially supported by the French National Research Agency, ANR (ASPEN Project), LabEx Plas@Par, and the French-Russian international laboratory LIA KaPPA 'Kinetics and Physics of Pulsed Plasmas and their Afterglow' (including RFBR project 17-52-16001 and CNRS financial and organizational support). The authors are thankful to Ali Mahjoub, Bruno Dufour and Pascal Pariset for technical assistance, and to Dr. Cyril Drag and Dr. Jean Paul Booth for the use of equipment essential for conducting this study. Useful discussions with Dr. Leanne C. Pitchford on $\mathrm{N}_{2}$ cross-sections, and with Prof. Igor V. Adamovich (from the Ohio State University) on the manuscript are also gratefully acknowledged. 


\section{References}

1. Samimy, M., Webb, N. and Esfahani, A., 2019. Reinventing the wheel: excitation of flow instabilities for active flow control using plasma actuators. Journal of Physics D: Applied Physics, 52(35), p.354002.

2. Leonov, S.B., Adamovich, I.V. and Soloviev, V.R., 2016. Dynamics of near-surface electric discharges and mechanisms of their interaction with the airflow. Plasma Sources Science and Technology, 25(6), p.063001.

3. Ju, Y. and Sun, W., 2015. Plasma assisted combustion: Dynamics and chemistry. Progress in Energy and Combustion Science, 48, pp.21-83.

4. Starikovskiy, A. and Aleksandrov, N., 2013. Plasma-assisted ignition and combustion. Progress in Energy and Combustion Science, 39(1), pp.61-110.

5. Starikovskaia, S.M., 2006. Plasma assisted ignition and combustion. Journal of Physics D: Applied Physics, 39(16), p.R265.

6. Pointu, A.M., Ricard, A., Odic, E. and Ganciu, M., 2008. Nitrogen atmospheric pressure post discharges for surface biological decontamination inside small diameter tubes. Plasma Processes and Polymers, 5(6), pp.559-568.

7. Duchesne, C., Banzet, S., Lataillade, J.J., Rousseau, A. and Frescaline, N., 2019. Cold atmospheric plasma modulates endothelial nitric oxide synthase signalling and enhances burn wound neovascularisation. The Journal of pathology.

8. Lagarkov, A.N. and Rutkevich, I.M., 2012. Ionization waves in electrical breakdown of gases. Springer Science \& Business Media.

9. Starikovskaia, S.M., Anikin, N.B., Pancheshnyi, S.V., Zatsepin, D.V. and Starikovskii, A.Y., 2001. Pulsed breakdown at high overvoltage: development, propagation and energy branching. Plasma Sources Science and Technology, 10(2), p.344.

10. Anikin, N.B., Zavialova, N.A., Starikovskaia, S.M. and Starikovskii, A.Y., 2008. Nanoseconddischarge development in long tubes. IEEE Transactions on Plasma Science, 36(4), pp.902903.

11. Brisset, A., Gazeli, K., Magne, L., Pasquiers, S., Jeanney, P., Marode, E. and Tardiveau, P., 2019. Modification of the electric field distribution in a diffuse streamer-induced discharge under extreme overvoltage. Plasma Sources Science and Technology, 28(5), p.055016.

12. Chng, T. L., Brisset, A., Jeanney, P., Starikovskaia, S.M., Adamovich, I. V. and Tardiveau, P., 2019. Electric Field Evolution in a Diffuse Ionization Wave Nanosecond Pulse Discharge in Atmospheric Pressure Air. Plasma Sources Science and Technology.

13. Aleksandrov, N. L. and Son, E. E., 1980. Chemistry of Plasma, ed. Smirnov BM (Moscow: Atomizdat), 7, p.35.

14. Stancu, G.D., Kaddouri, F., Lacoste, D.A. and Laux, C.O., 2010. Atmospheric pressure plasma diagnostics by OES, CRDS and TALIF. Journal of Physics D: Applied Physics, 43(12), p.124002.

15. Bak, M.S., Kim, W. and Cappelli, M.A., 2011. On the quenching of excited electronic states of molecular nitrogen in nanosecond pulsed discharges in atmospheric pressure air. Applied Physics Letters, 98(1), p.011502.

16. Rusterholtz, D.L., Lacoste, D.A., Stancu, G.D., Pai, D.Z. and Laux, C.O., 2013. Ultrafast 
heating and oxygen dissociation in atmospheric pressure air by nanosecond repetitively pulsed discharges. Journal of Physics D: Applied Physics, 46(46), p.464010.

17. Lo, A., Cessou, A., Boubert, P. and Vervisch, P., 2014. Space and time analysis of the nanosecond scale discharges in atmospheric pressure air: I. Gas temperature and vibrational distribution function of $\mathrm{N}_{2}$ and $\mathrm{O}_{2}$. Journal of Physics D: Applied Physics, 47(11), p.115201.

18. Klochko, A.V., Lemainque, J., Booth, J.P. and Starikovskaia, S.M., 2015. TALIF measurements of oxygen atom density in the afterglow of a capillary nanosecond discharge. Plasma Sources Science and Technology, 24(2), p.025010.

19. Popov, N.A., 2011. Fast gas heating in a nitrogen-oxygen discharge plasma: I. Kinetic mechanism. Journal of Physics D: Applied Physics, 44(28), p.285201.

20. Lepikhin, N.D., Popov, N.A. and Starikovskaia, S.M., 2018. Fast gas heating and radial distribution of active species in nanosecond capillary discharge in pure nitrogen and $\mathrm{N}_{2}: \mathrm{O}_{2}$ mixtures. Plasma Sources Science and Technology, 27(5), p.055005.

21. Klochko, A.V., Starikovskaia, S.M., Xiong, Z. and Kushner, M.J., 2014. Investigation of capillary nanosecond discharges in air at moderate pressure: comparison of experiments and 2D numerical modelling. Journal of Physics D: Applied Physics, 47(36), p.365202.

22. Anikin, N.B., Starikovskaia, S.M. and Starikovskii, A.Y., 2004. Study of the oxidation of alkanes in their mixtures with oxygen and air under the action of a pulsed volume nanosecond discharge. Plasma physics reports, 30(12), pp.1028-1042.

23. Kramida, A., Ralchenko, Y. and Reader, J., 2018. NAT NIST atomic spectra database (version 5.5.6).

24. Niemi, K., Schulz-Von Der Gathen, V. and Döbele, H.F., 2001. Absolute calibration of atomic density measurements by laser-induced fluorescence spectroscopy with two-photon excitation. Journal of Physics D: Applied Physics, 34(15), p.2330.

25. Wang, Y., Capps, C. and Kulatilaka, W.D., 2017. Femtosecond two-photon laser-induced fluorescence of krypton for high-speed flow imaging. Optics letters, 42(4), pp.711-714.

26. Dumitrache, C., Gallant, A., Stancu, G.D. and Laux, C.O., 2019. Femtosecond Two-Photon Absorption Laser Induced Fluorescence (fs-TALIF) Imaging of Atomic Nitrogen in Nanosecond Repetitive Discharges. In AIAA Scitech 2019 Forum (p. 1507).

27. Lepikhin, N.D., Klochko, A.V., Popov, N.A. and Starikovskaia, S.M., 2016. Long-lived plasma and fast quenching of $\mathrm{N}_{2}\left(\mathrm{C}^{3} \Pi_{\mathrm{u}}\right)$ by electrons in the afterglow of a nanosecond capillary discharge in nitrogen. Plasma Sources Science and Technology, 25(4), p.045003.

28. Adams, S.F. and Miller, T.A., 1998. Two-photon absorption laser-induced fluorescence of atomic nitrogen by an alternative excitation scheme. Chemical physics letters, 295(4), pp.305311.

29. Cosby, P.C., 1993. Electron-impact dissociation of nitrogen. The Journal of chemical physics, 98(12), pp.9544-9553.

30. Popov, N.A., 2013. Dissociation of nitrogen in a pulse-periodic dielectric barrier discharge at atmospheric pressure. Plasma Physics Reports, 39(5), pp.420-424.

31. Galvão, B.R.L., Varandas, A.J.C., Braga, J.P. and Belchior, J.C., 2013. Electronic quenching of $\mathrm{N}\left({ }^{2} \mathrm{D}\right)$ by $\mathrm{N}_{2}$ : theoretical predictions, comparison with experimental rate constants, and impact on atmospheric modeling. The Journal of Physical Chemistry Letters, 4(14), pp.2292- 
2297.

32. Raizer, Y.P., 1991. Gas discharge physics.

33. Capitelli, M., Ferreira, C.M., Gordiets, B.F. and Osipov, A.I., 2013. Plasma kinetics in atmospheric gases (Vol. 31). Springer Science \& Business Media.

34. Marshall, T., 1962. Surface recombination of nitrogen atoms upon quartz. The Journal of Chemical Physics, 37(10), pp.2501-2502.

35. Czerwiec, T., Greer, F. and Graves, D.B., 2005. Nitrogen dissociation in a low pressure cylindrical ICP discharge studied by actinometry and mass spectrometry. Journal of Physics D: Applied Physics, 38(24), p.4278.

36. Dvorak, P., Šimek, M. and Prukner, V., 2019. Evolution of $\mathrm{N}\left({ }^{4} \mathrm{~S}\right)$ atoms produced under nitrogen streamer conditions: time-resolved TALIF study at reduced pressures. Plasma Sources Science and Technology, 28, p.125004.

37. Es-Sebbar, E.T., Sarra-Bournet, C., Naudé, N., Massines, F. and Gherardi, N., 2009. Absolute nitrogen atom density measurements by two-photon laser-induced fluorescence spectroscopy in atmospheric pressure dielectric barrier discharges of pure nitrogen. Journal of Applied Physics, 106(7), p.073302.

38. Fresnet, F., Baravian, G., Pasquiers, S., Postel, C., Puech, V., Rousseau, A. and Rozoy, M., 2000. Time-resolved laser-induced fluorescence study of NO removal plasma technology in $\mathrm{N}_{2}$ /NO mixtures. Journal of Physics D: Applied Physics, 33(11), p.1315.

39. Hagelaar, G.J.M. and Pitchford, L.C., 2005. Solving the Boltzmann equation to obtain electron transport coefficients and rate coefficients for fluid models. Plasma Sources Science and Technology, 14(4), p.722.

40. 2013 SIGLO database http://www.lxcat.laplace.univ-tlse.fr [2013, June 4].

41. Bacri, J. and Medani, A., 1982. Electron diatomic molecule weighted total cross section calculation: III. Main inelastic processes for $\mathrm{N}_{2}$ and $\mathrm{N}_{2}{ }^{+}$. Physica B+ C, 112(1), pp.101-118.

42. Phelps, A.V. and Pitchford, L.C., 1985. Anisotropic scattering of electrons by $\mathrm{N}_{2}$ and its effect on electron transport. Physical Review A, 31(5), p.2932. 